\title{
Les pôles dans leur espace périphérique
}

\author{
Marc-Urbain Proulx ${ }^{a}$
}

RÉSUMÉ. La ruralité québécoise nécessite des observations pointues pour saisir et analyser sa complexité. Aussi, un regard géoéconomique global offre des perspectives utiles pour éclairer certains phénomènes, notamment la présence de pôles hiérarchisés au sein d'un espace mouvant à mieux maitriser dans ses tendances, telles que l'attraction, la concentration, l'érosion, la rétention, la répulsion, la pénétration et la diffusion.

ABSTRACT. Quebec's rurality requires sharp observations to grasp and analyze its complexity. A global geo-economic look also offers useful perspectives to shed light on certain phenomena, notably the presence of hierarcbical poles within a moving space to better control its tendencies such as attraction, concentration, erosion, retention, repulsion, penetration and diffusion.

Selon une analyse géoéconomique globale, le Québec apparait comme une grande région excentrée au nord-est de son continent. Puisque le centre de gravité de la population continentale se déplace lentement vers l'ouest (Tellier, 1996), tiré dans cette mouvance spatiale par la polarisation économique mondiale des dragons asiatiques, le Québec voit en conséquence s'accentuer son caractère périphérique.

Localisée au sud de cette périphérie nord-américaine, la métropole Montréal, qui a déclassé Québec en bénéficiant de l'industrialisation impulsée par la canalisation du fleuve Saint-Laurent au milieu du XIX ${ }^{\mathrm{e}}$ siècle, fut à son tour déclassée par l'émergente Toronto, certes mieux positionnée pour son intégration comme capitale canadienne dans le système urbain continental. Depuis les années 1950, la Ville Reine domine de plus en plus l'urbanisation canadienne en polarisant largement les activités du tertiaire supérieur (finance, sièges sociaux, services spécialisés, équipements culturels, etc.), au détriment de Montréal, qui, de 1850 à 1960, fut en explosion dans ce secteur névralgique, qui appartenait auparavant à Québec.

Parmi les causes du déclin relatif de Montréal (Polèse, 1990), qui semble s'atténuer récemment, nul doute que l'ouverture de la voie maritime du Saint-Laurent jusqu'aux Grands Lacs en 1959 fut déterminante. Le drainage accentué de la richesse et des ressources du
Québec vers Toronto fut évident, malgré les bons instruments de rétention mis en œuvre, dont les diverses sociétés d'État. Devenu secondaire et concurrencé dans cette catégorie par entre autres les villes d'Ottawa, de Calgary et de Moncton, le pôle Montréal peine désormais à jouer son rôle de moteur économique du Québec.

\section{Québec central}

Comme partout au Canada, la frontière québécoise qui donne accès aux grands marchés américains représente un important facteur d'attraction des activités et de la population. Cette accessibilité explique largement la croissance de Montréal au cours des deux derniers siècles. Elle explique aussi la bonne performance économique actuelle des régions centrales de la Montérégie, de l'Estrie, des Bois-Francs, de la Beauce et des Laurentides.

Au sein de cette vaste zone «métapolitaine» (voir figure 1) incluant une large couronne périurbaine densifiée autour des principaux axes de transport, deux autres phénomènes territoriaux différenciés s'affirment clairement (Proulx, 2019). Il s'agit d'abord de l'anneau manufacturier formé par les villes de Victoriaville, de Sherbrooke, de Granby, de SaintHyacinthe et de Drummondville. Ces pôles illustrent actuellement les taux de croissance de l'emploi les plus élevés du Québec. Il s'agit en ces lieux d'une localisation manufacturière optimale. Entre cette couronne

${ }^{a}$ Directeur scientifique du CRDT et professeur, Université du Québec à Chicoutimi 
et le corridor beauceron, bien connu pour le dynamisme de ses PME manufacturières, se présente une enclave territoriale très peu industrialisée puisque mal reliée au réseau de transport conduisant aux grands marchés américains, y compris Montréal.

Notre analyse nous permet aussi, en substance, de confirmer l'importance du fleuve Saint-Laurent dans la localisation des activités industrielles. Signalons qu'en matière de transport, le mode routier a supplanté celui du maritime au cours des dernières décennies. Cela rend moins attrayants les lieux positionnés sur les rives du fleuve. En conséquence, la zone portuaire planifiée de Bécancour, près du fleuve, s'est fait déclasser par le carrefour industriel de Drummondville, devenu très attrayant.

En réalité, mises à part quelques petites poches d'activités dans Lanaudière et dans l'aire métropolitaine de Québec, le secteur manufacturier se localise que très peu au nord du fleuve. S'accroissant avec la distance de la frontière américaine, les couts de transport sont proposés comme un facteur explicatif de cette répulsion territoriale. Aussi, au nord du fleuve, les salaires versés par les grandes entreprises extractives de ressources naturelles, qui font pression à la hausse sur les couts de main-d'œuvre, deviennent dissuasifs pour les manufactures (Polèse et Shermur, 2002).

Si la Basse-Mauricie, le Saguenay et la zone d'Amqui font légèrement exception à cette règle de la localisation industrielle grâce à leur marché local plus important, les régions nordiques de la Haute-Mauricie, de l'Outaouais hors Gatineau, de la Côte-Nord, du LacSaint-Jean et de l'Abitibi-Témiscamingue se comportent comme la Gaspésie, avec des structures économiques primaires peu structurantes des filières industrielles (forêt, mines, pêches, etc.). Leur industrialisation ne franchit pas, ou si peu, l'étape de la maturité associée à la diversification économique, notamment par la transformation des matières premières, qui demeurent hélas largement expédiées à l'état brut, sans beaucoup de valeur ajoutée (Proulx, 2011). Dans ces régions périphériques, l'érosion hors des circuits économiques de la richesse créée avec les ressources naturelles s'avère fort importante. Ces régions sont en conséquence largement dépendantes des impulsions exogènes (grands projets) reliées à l'établissement d'activités extractives de ressources naturelles.

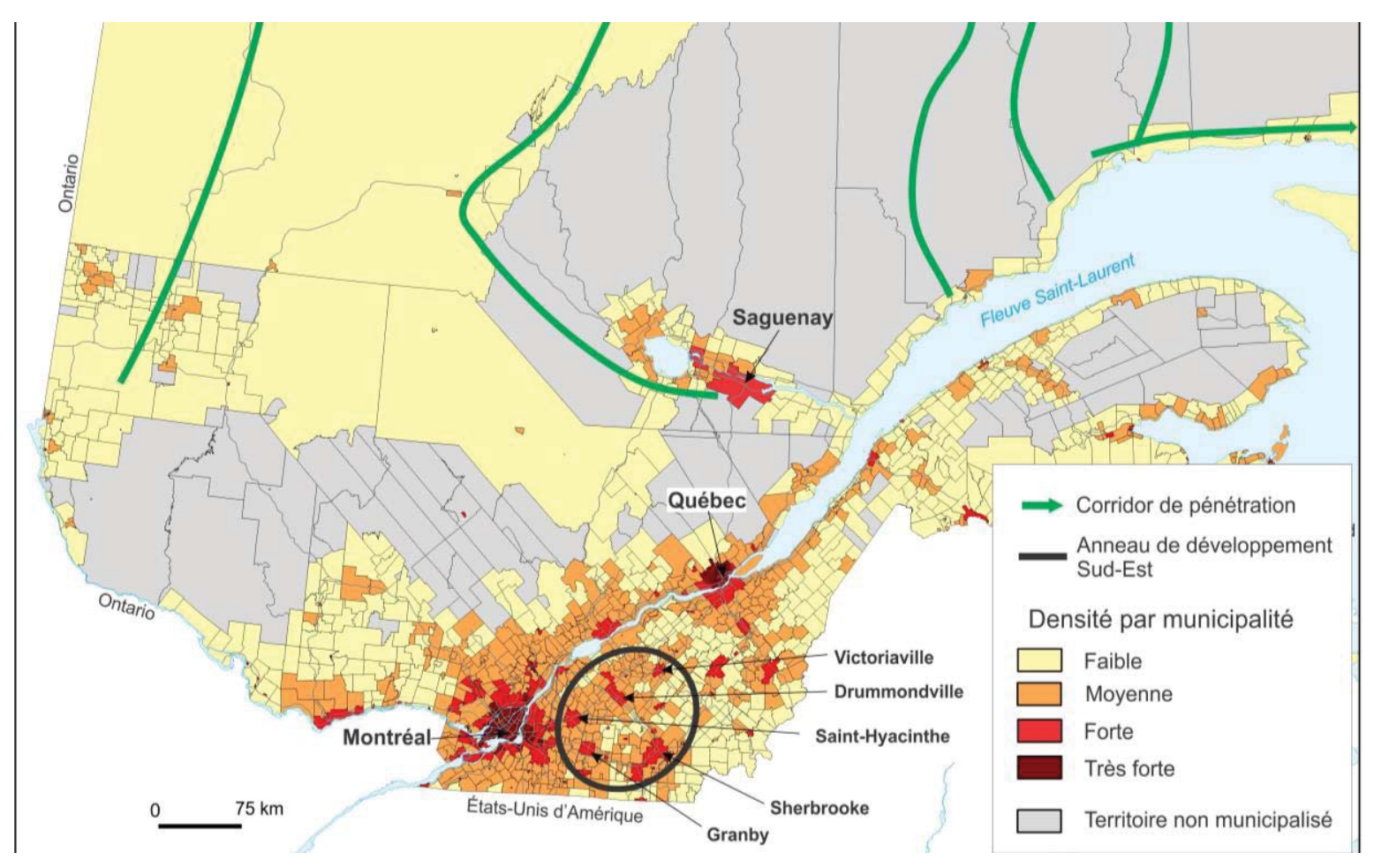

Figure 1 - Formes territoriales du Québec contemporain Source : Statistique Canada, Carl Brisson, LERGA, UQAC 


\section{Avant-postes}

On sait que l'espace Québec, à $85 \%$ rural en 1850, est devenu urbain à $80 \%$ en 1966. Bien sûr, ce mouvement d'urbanisation intensive a beaucoup transformé l'ensemble du monde rural (Fortin, 1971), mais aussi toute la structure de peuplement (La Haye, 1968). Au cours de la décennie 1960, caractérisée par des questionnements nationaux systématiques, notamment à propos de l'aménagement des territoires, une étude gouvernementale a pertinemment illustré le système urbain du Québec, composé de pôles primaires, secondaires, tertiaires et quaternaires (Proulx, 2006), afin de pointer les enjeux dans un esprit d'une meilleure maitrise des tendances spatiales.

Si le rapport Higgins, Martin et Raynauld (HMR, 1970) a statué sur l'importance de cibler les interventions publiques structurantes à Montréal afin que les effets de diffusion de son développement influencent tout le Québec, il demeure que cette hiérarchisation bien illustrée des centres urbains offrait au gouvernement une solide logique pour ordonnancer la répartition équitable des équipements et des services publics en éducation, en santé, en transport, en loisirs, en services sociaux, etc. Cette modélisation globale de l'armature urbaine dispersée à travers l'espace à aménager permettait aussi de mieux comprendre les forces, les contraintes et les occasions qui structurent les territoires en illustrant quelques fois des menaces, dont l'érosion rurale, l'étalement urbain et la dévitalisation de zones centrales. Aussi, il fut bien compris que la localisation spécifique d'un lieu n'était pas le fruit du hasard, mais représentait plutôt un solide déterminant de sa croissance.

À cet effet, si la dotation en ressources naturelles occasionne l'établissement de lieux éloignés et dispersés en périphérie, la nécessité de transbordements des matières et de transits de la maind'œuvre explique la polarisation de certaines activités à Sept-Îles, Rivière-du-Loup, Baie-Comeau, Amos, Saguenay, Rimouski, Forestville et SaintFélicien (Proulx, 2002, 2011). Dans l'armature urbaine du Québec, ces pôles en région s'inscrivent tels des avant-postes de pénétration périphérique vers les bassins de ressources. Il convient d'en comprendre le fonctionnement.
En ces lieux, la concentration de commerces de gros, de constructeurs, de services publics, d'administrations privées et publique, de services spécialisés, d'usines pour l'affinage, de fabriques d'équipements et de biens, de fournisseurs divers et de groupes de la société civile s'avère largement reliée aux activités et à la population présentes dans leur zone de rayonnement accessible par des corridors de transport (voir figure 1). En offrant les infrastructures et équipements pour la livraison de quantité souvent phénoménale de matières premières sur le marché mondial, ces pôles permettent aussi, pertinemment, la capacité de rétention territoriale d'une certaine partie de la rente régionale de natures forestière, minière, hydroélectrique, agricole et maritime. Si des outils de maximisation de cette rétention sont déjà présents à cet effet, d'autres sont à inventer impérativement.

Signalons qu'au contraire d'une rétention accrue actuelle, force est de constater un accroissement des fuites financières hors des circuits régionaux (Proulx, 2014a). Deux causes expliquent ce fléau. D’abord, nous assistons au remplacement généralisé de nombreux postes de travail par des technologies sophistiquées au sein des activités d'extraction et de première transformation. Les emplois sont plus qualitatifs, certes, mais aussi beaucoup moins nombreux. À titre d'exemple, le nombre de travailleurs pour extraire 1 million de tonnes de fer dans les mines du Québec était de 459 en 1950, mais de 153 en 2010 (soit trois fois moins). Il sera de 51 en 2020 (Proulx, 2019), soit une autre division par trois des emplois pour la même quantité expédiée de minerais.

Ensuite, les emplois tout de même encore créés dans l'extraction sont de plus en plus pourvus par des travailleurs navetteurs (fly-in fly-out workers), qui viennent largement des grands centres urbains de Montréal et de Québec, où sont affrétés les avions navetteurs. S'avère aussi révolue l'époque de l'émergence de villes champignons (boom towns) pour loger les travailleurs et leur famille autour des bassins de ressources nouvellement exploitées en périphérie du Québec. En conséquence de ce peu d'emplois pourvus par des travailleurs locaux ou régionaux, de nombreux villages et petites villes localisés en périphérie sont devenus déclinants ou stagnants, puis menacent le niveau et la qualité des biens et services publics desservis. 
Pour tenter de colmater cette perte régionale bien perçue, une stratégie gouvernementale de la transformation des matières premières fut mise sur pied. Malgré les importantes mesures incitatives de la politique publique des dernières décennies, qui a ciblé le financement du risque, les crédits d'impôt, l'entrepreneuriat, la formation de main-d'œuvre et l'animation socioéconomique, cette stratégie n'a cependant généré que des résultats modestes en région (Proulx, 2011, 2019). Récemment, la désignation des technopoles (recherche, R-D, transferts technologiques) autour des universités régionales (dans l'énergie en Mauricie, le maritime à Rimouski, l'aluminium à Saguenay et les mines à Rouyn) représente la volonté du stratège de miser sur des spécialisations sectorielles. Les effets structurants sont réels, mais aucunement impressionnants pour l'instant, pas même dans la production d'énergie éolienne, qui a nécessité des efforts publics pourtant considérables.

Force est de constater qu'en matière de transformation des ressources naturelles, les forces du marché ont la tête dure. Il apparait en réalité plus rentable pour les entreprises d'acheminer les matières premières à transformer vers les usines à grand gabarit localisées près des grands marchés de consommation de produits semi-finis et finis, notamment dans l'Est américain et le Midwest américain, très bien dotés de mesures incitatives et protectionnistes pour l'industrie. Néanmoins, cette absence de valeur ajoutée sur les lieux d'extraction n'empêche pas les extracteurs de ressources naturelles de quémander l'aide de l'État québécois pour soutenir davantage leur profitabilité. Le très actuel Plan Nord s'avère très révélateur à cet égard (Proulx, 2014a).

\section{Consolidation publique}

En matière de rétention supplémentaire de la rente régionale en périphérie, les leviers économiques efficaces sont plutôt rares. En réalité, au Québec, les véritables instruments économiques tels qu'Hydro-Québec, la Caisse de dépôt, Investissement Québec et autres sociétés d'État s'inscrivent à l'échelle nationale avec certes un rayonnement régional. Ils limitent avec bonheur l'érosion économique hors Québec, mais ne contraignent que très peu le drainage des régions (Prémont et Proulx, 2020). On a jadis misé sur le régionalisme. À cet effet, si cinq décennies de planification régionale ont certes permis de rationaliser considérablement les prises de décisions, les régions n'ont pas réussi à établir de véritables leviers économiques durables, si ce n'est l'historique système coopératif (Proulx, 2019).

On a par contre ciblé le renforcement des principaux pôles urbains en établissant des équipements (santé, éducation, loisirs, culture, etc.), des infrastructures de transport et aussi des agences publiques associées aux diverses fonctions exercées de manière déconcentrée ou décentralisée par les gouvernements supérieurs. Puisqu'ils sont garants en région d'un cadre de vie de qualité et d'un bon niveau de services publics, ces pôles multifonctionnels représentent un atout considérable.

En 2002, la plupart des avant-postes établis en région ont à cet effet bénéficié de la fusion municipale des agglomérations urbaines. La réorganisation des municipalités du Québec visait la consolidation des pôles québécois. Des gains d'efficacité furent obtenus dans la gestion publique municipale de biens et services collectifs.

Au sein de ces pôles, à cet effet, il existe un important secteur public non municipal composé d'agences gouvernementales autonomes des municipalités qui exercent dans la santé, l'éducation, l'emploi, la sécurité publique, le transport et le développement économique en utilisant la planification stratégique et les plans d'action. Il représente en général une masse de ressources financières allouées bien supérieure à celle du secteur public municipalisé.

À Saguenay, à titre d'exemple, moins de $25 \%$ du budget annuel de fonctionnement du secteur public local est attribuable à la municipalité (Proulx, 2007). Nul doute qu'une seconde phase de la réorganisation municipale engagée en 2002 pourrait viser l'intensification de la collaboration entre toutes ces administrations municipales et non municipales, selon une vision globale du secteur public local à consolider, dans le respect de l'autonomie de chacun.

Il existe plusieurs cas de succès au Québec (Proulx, 2014b), notamment Sherbrooke et Laval. Sur cette base, une stratégie gouvernementale explicite et bien ciblée serait bénéfique. Cela pourrait assurément générer des synergies supplémentaires et, en conséquence, renforcer la capacité collective de prise en main de leviers concernant la valorisation 
du bien commun au sein des avant-postes de pénétration en périphérie.

À cet effet de consolidation publique, le spécialiste Clermont Dugas (2012, 2019) propose pertinemment les conditions d'une stratégie rurale basée sur le renforcement des petits centres de services bien positionnés en leur offrant un rôle revalorisé comme complément significatif dans l'armature urbaine du Québec.

D'une importance majeure pour le monde rural en périphérie, ces établissements humains, répartis au sein de 65 territoires MRC, sont déjà pour la plupart plus ou moins en cours de consolidation. Ils témoignent de la présence de forces et d'avantages spécifiques qui leur offrent un potentiel particulier. Ce sont des pôles tertiaires de rétention de la population rurale en érosion.

En ciblant quelques enjeux concrets, dont la déconcentration administrative, l'entrepreneuriat local, l'aménagement du territoire ainsi que l'offre de nouveaux équipements et services, le professeur Dugas propose des critères ancrés dans la réalité contemporaine pour la sélection des centres de services à cibler, notamment la localisation, la taille, les habitudes de mobilité, la masse critique de facteurs déjà présents et la distance. Il reste maintenant à élaborer une stratégie rurale conséquente, capable d'effectuer des choix sur des cibles, plutôt que de disperser les efforts tous azimuts. À cet effet, les aires de rayonnement périphérique de ces pôles ruraux ont aussi la responsabilité de la protection et de la défense de leur moteur de développement territorial de natures sociale, économique et culturelle.

\section{Conclusion}

Pour assurer l'avenir de la ruralité en périphérie dans le contexte du déclin démographique actuel causé par les pertes massives d'emplois dans les secteurs économiques traditionnels, nul doute que la solution optimale résiderait dans l'obligation d'imposer aux extracteurs d'ajouter de la valeur aux matières premières avant l'expédition afin de créer de l'emploi et de la richesse sur place. Pour justifier cette obligation non incluse dans les nouveaux régimes forestiers et miniers québécois, il s'agirait d'utiliser l'analyse coutavantage comme argument de base.

Puisque cette solution semble difficile à mettre en œuvre par nos gouvernements insuffisamment instrumentés pour faire face à l'industrie de l'extraction, il faut miser sur des leviers mieux maitrisables. La logique des établissements humains illustre le phénomène de polarisation comme un atout à valoriser, notamment au sein des avant-postes de pénétration périphérique et des petits centres ruraux.

Dans ces deux types de pôles, il existe un important potentiel de synergie territoriale insuffisamment exploitée par un secteur public fragmenté par des agences publiques municipales et non municipales autonomes, indépendantes et même en concurrence sur certains aspects. Une stratégie gouvernementale de renforcement de ces pôles par la coordination des fonctions publiques exercées représente une piste de solution pertinente.

\section{RÉFÉRENCES}

Dugas, C. (2002). Une stratégie de renforcement des petits centres ruraux. Organisations \& Territoires, 11(1), 11-16. https://doi.org/10.1522/revueot.v11n1.820

Dugas, C. (2019). Le renforcement des centres de services : un moyen d'atténuation des disparités territoriales. Dans M.-U. Proulx et M.-C. Prémont (dir.), La politique territoriale au Québec (p. 273-302). Québec, QC: Presses de l'Université du Québec.

Fortin, G. (1971). La fin d'un règne. Montréal, QC : HMH.

HMR (1970). Les orientations du développement économique régional dans la province de Québec, Rapport Higgins, B., Martin, F. et Raynauld, A., Ottawa, Ministère de l'Expansion économique régionale.

La Haye, J.-C. (1968). Rapport de la commission provinciale d'urbanisme. Québec, QC : Imprimeur de la Reine.

Polèse, M. (1990). La thèse du déclin de Montréal, revue et corrigée. L'Actualité économique, 66(2), 133-146. https://doi.org/10.7202/601525ar 
Polèse, M. et Shearmur, R. (2002). La périphérie face à l'économie du savoir. Montréal, QC : INRS-Urbanisation, Culture et Société/Institut canadien de recherche sur le développement régional.

Prémont, M.-C et Proulx, M.-U. (2020). L’hydroélectricité et les grandes régions productrices, Organisations er Territoires, 29(1).

Proulx, M.-U. (2002). L'économie des territoires an Québec. Québec, QC : Presses de l’Université du Québec.

Proulx, M.-U. (2006). La mouvance contemporaine des territoires : la logique spatiale de l'économie au Québec. Recherches sociographiques, 47(3), 475-502. https://doi.org/10.7202/014655ar

Proulx, M.-U. (2007). Vision 2025 : le Saguenay-Lac-Saint-Jean face à son avenir. Québec, QC : Presses de l’Université du Québec.

Proulx, M.-U. (2011). Territoires et développement : la richesse du Québec. Québec, QC : Presses de l’Université du Québec.

Proulx, M.-U. (2012). Centre et périphérie : le nouveau modèle québécois. Revue d'économie régionale et urbaine, 4, 597-622. https://doi.org/10.3917/reru.124.0597

Proulx, M.-U. (2014a). Nouveau cycle économique en périphérie nordique. L'Actualité économique, 90(2), 121-144. https://doi.org/10.7202/1027975ar

Proulx, M.-U. (2014b. Territoires MRC du Québec : planification et gouvernance d'État. Économie et solidarité, 44(1-2). https://doi.org/10.7202/1041602ar

Proulx, M.-U. (2019). Splendeurs, misères et ressorts des régions. Québec, QC : Presses de l’Université du Québec.

Tellier, L.-N. (1996). Le Québec et ses régions à l'intérieur de la dynamique spatiale de l'économie mondiale. Dans M.-U. Proulx (dir.), Le phénomène régional au Québec (p. 9-28). Québec, QC : Presses de l'Université du Québec. 